\title{
FLUOROSCOPY DURATION IN ORTHOPEDIC SURGERY
}

João Caron La Salvia', Pablo Reis de Moraes², Tiago Yossef Ammar ${ }^{3}$, Carlos Roberto Schwartsmann ${ }^{4}$

\section{ABSTRACT}

Objective: To ascertain the mean length of radiation emission from fluoroscopic devices during several types of orthopedic surgery and which of these required greater use of radiation. Methods: The times taken to perform sixteen different types of surgery (total of 80 procedures) were measured. At the end of each procedure, the length of time for which fluoroscopy was used directly from the image intensifier was ascertained. Results: The mean time required for fluoroscopy per operation was 61 seconds. The procedures that demanded greatest mean duration of radiation use were bilateral proximal femoral epiphysiodesis (5.1 minutes) and femoral shaft osteosynthesis using a locked intramedullary nail (3.33 $\mathrm{min})$. Conclusion: The mean duration of fluoroscopy use in orthopedic operations was 61 seconds. The procedures using an intramedullary device were the ones that required greatest radiation emission.

Keywords - Radiation Dosage; Fluoroscopy; Orthopedics

\section{INTRODUCTION}

The harmful effects of high doses of radiation in humans are well-known, including cell death and increased incidence of cancer, among others. The effects of low doses of radiation in the long term have still not been clearly established by studies on humans ${ }^{(1,2)}$. In medical practice, however, it is assumed that these risks exist, and preventative measures are taken to prevent or minimize them.

Thousands of orthopedic surgeries are carried out each year. Many of these require continuous use of fluoroscopy. With the advance in percutaneous techniques, locked intramedullary devices, the increasing use of radiation during these operations is noted, causing concern as to the possible harmful effects for the patient and the medical team. It is known that a fluoroscopy device can emit $0.4 \mathrm{rad} / \mathrm{min}$, depending largely on its calibration. The majority of the US guidelines suggest an annual limit of exposure to radiation of not more than $5 \mathrm{rad}^{(1)}$.

However, the real time that the medical team and patients are exposed to radiation during the various orthopedic surgeries is not known. It is also not known which operations require more radiation emission time.

Seeking to resolve these questions, the fluoroscopy usage times were determined for 80 orthopedic surgeries performed at the Sarmento Barata Surgical Center of the Hospital Santa Clara (Complexo Hospitalar Santa Casa de Porto Alegre).

\section{MATERIAL AND METHODS}

From March to August 2009, the durations of 80 orthopedic surgeries performed at the abovementioned hospital were determined. All the surgical procedures were carried out using the same mobile image intensifier (Philips Endura).

1 - Resident Doctor of the Orthopedics and Traumatology Service of the Complexo Hospitalar Santa Casa de Porto Alegre, RS, Brazil.

2 - Medical Student in the $6^{\text {th }}$ year at the Universidade Federal de Rio Grande, Porto Alegre, RS, Brazil.

3 - Medical Student in the $6^{\text {th }}$ year at the Universidade Federal de Ciências da Saúde de Porto Alegre, Porto Alegre, RS, Brazil.

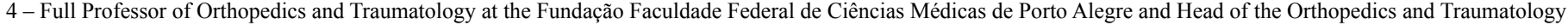
Service of the Complexo Hospitalar Santa Casa de Porto Alegre, RS, Brazil. 
In order to determine as closely as possible the actual mean time, the surgeries were performed by various surgeons, some more experienced some less so, including resident doctors in training in Orthopedics and Traumatology at this service, working under supervision.

No preference was given to any specific surgical procedure, and the surgeries were randomly selected. Sixteen different operations were analyzed, totaling 80 procedures: hip arthroscopy (four procedures); bilateral proximal femoral epiphysiodesis - cannulated screws (one); hallux valgus correction (eight); varization osteotomy of the femur (two); valgisation osteotomy of the tibia (two); osteosynthesis of the metacarpal bones - intramedullary wire fixation (seven); osteosynthesis of fracture of the distal third of the radius - plate and screws (12) - percutaneous Kirschner wires (four); osteosynthesis of fracture of the neck of the femur - canulated screws (three); osteosynthesis of transtrochanteric fracture - DHS plate (four); osteosynthesis of the scaphoid - self-compressing screw (six); osteosynthesis of the proximal humerus - angled plate (five); osteosynthesis in diaphysiary fractures of the femur - locked intramedullary nail (nine); osteosynthesis in diaphysiary fractures of the tibia - locked intramedullary nail (five); osteosynthesis of the proximal tibia - plate and screws (two); osteosynthesis of ankle fracture - plate and screws (six).

The duration was recorded immediately after each surgical procedure. The value was obtained directly from the display of the image intensifier, and reflects the exact time of radiation emitted in each procedure.

\section{RESULTS}

Of the 80 surgical procedures performed, 44 (55\%) were in male patients and $36(45 \%)$ were in female patients. The average age of the patients was 44 years (9-91 years).

The total fluoroscopy time used in the 80 surgical procedures was 4897 seconds (1 hour, 20 minutes and 31 seconds). The average was 61 seconds per procedure.

The result of our study is shown in Table 1. It is emphasized that the operations that require greater use of radiation were those which used locking intramedullary device. We used, on average, 200 seconds to perform osteosynthesis of diaphysiary fracture of the femur with locked intramedullary nail (LIN) and 102 seconds to perform osteosynthesis of diaphysiary fracture of the tibia with LIN. The only case of proximal femoral epiphysiodesis with canulated screws also required significant use of radiation (310 seconds). Also of note is the low requirement for fluoroscopy in percutaneous synthesis of fractures of the neck of the femur with the use of canulated screws (less than one second of radiation emission per procedure).

Also of note is the wide disparity in fluoroscopy times within a single procedure. During the femoral

Table 1 - List of surgeries, with fluoroscopy times.

\begin{tabular}{|c|c|c|c|c|c|}
\hline \multirow{2}{*}{ Surgery } & \multirow[t]{2}{*}{ QTY } & \multicolumn{4}{|c|}{ Time } \\
\hline & & Medium & Minimum & Maximum & Total \\
\hline Hip arthroscopy & 4 & 63.00 & 18.00 & 150.00 & 252.00 \\
\hline Proximal femoral epiphysiodesis & 1 & 310.80 & 310.80 & 310.80 & 310.80 \\
\hline Hallux Valgus & 8 & 13.70 & 0.60 & 45.00 & 109.60 \\
\hline Femur LIN ${ }^{1}$ & 9 & 200.44 & 65.00 & 516.00 & 1804.00 \\
\hline Tibia LIN ${ }^{1}$ & 5 & 102.35 & 2.36 & 205.00 & 511.76 \\
\hline Osteosynthesis neck of femur canulated screw & 3 & 0.48 & 0.35 & 0.57 & 1.44 \\
\hline Osteosynthesis scafoid & 6 & 32.96 & 0.38 & 120.00 & 197.74 \\
\hline Osteosynthesis metacarpal bones & 7 & 29.93 & 0.30 & 127.20 & 209.50 \\
\hline Osteosynthesis tibial plate ORIF $^{2}$ & 2 & 15.65 & 1.30 & 30.00 & 31.30 \\
\hline Osteosynthesis distal third of radius CRPP $^{3}$ & 4 & 24.30 & 2.18 & 39.00 & 97.18 \\
\hline Osteosynthesis distal third of radius volar plate & 12 & 41.04 & 0.53 & 120.00 & 492.53 \\
\hline Osteosynthesis ankle & 6 & 45.25 & 0.50 & 124.00 & 271.50 \\
\hline Osteosynthesis transtrochanteric femur & 4 & 60.00 & 50.00 & 64.00 & 240.00 \\
\hline Osteosynthesis proximal humerus & 5 & 42.24 & 1.20 & 78.00 & 211.20 \\
\hline Valgisation osteotomy tibia & 2 & 13.24 & 0.48 & 26.00 & 26.48 \\
\hline Varization osteotomy femur & 2 & 65.00 & 54.00 & 76.00 & 130.00 \\
\hline
\end{tabular}


osteosynthesis with LIN, the time ranged from 65 to 516 seconds. In osteosynthesis of fracture of the distal third of the radius with the use of plate and screws, the time ranged from less than one to 120 seconds.

\section{DISCUSSION}

The creation of a method for instant and dynamic radiological evaluation during the surgical procedures led to a change in surgical treatment of various diseases. Its use is considered almost indispensible in the majority of osteosynthesis procedures. We also observed that its use is increasing in elective orthopedic surgeries.

The risk of radiation and its association with cancer is well-known ${ }^{(3)}$. The effects of prolonged exposure to low doses of radiation are not known, and there is no known safe dose. An incidence of cancer in orthopedists four times higher than in specialists of other areas, and eight times higher than control workers not exposed to radiation, has been reported in the literature $^{(4)}$. There are basically three proven ways of decreasing this exposure ${ }^{(5)}$ : use of protective jackets and collars, increasing the distance from the emission source of radiation, and decreasing the exposure time.

There are few studies in the literature that investigate the duration of radiation emitted in orthopedic surgeries, however, there are studies with other purposes that cite the average fluoroscopy time in these operations. Tsalafoutas et $\mathrm{al}^{(6)}$ published an average of 90 seconds of fluoroscopy for malleolar fractures, 108 seconds for fractures of the distal third of the radius with use of plate, 378 seconds for locked intramedullary nail (LIN) of the femur, and 312 seconds for LIN

\section{REFERENCES}

\footnotetext{
1. Herscovici D Jr, Sanders RW. The effects, risks, and guidelines for radiation use in orthopaedic surgery.Clin Orthop Relat Res. 2000;(375):126-32.

2. Devalia KL, Peter VK, Madanur MA, Braithwaite IJ. Exposure of the thyroid to radiation during routine orthopaedic procedures. Acta Orthop Belg. 2006;72(5):615-20

3. Barry T. Radiation exposure to an orthopaedic surgeon. Clin Orthop Relat Res. 1984;(182):160-4

4. Mastrangelo G, Fedeli U, Fadda E, Giovanazzi A, Scoizzato L, Saia B. Increased cancer risk among surgeons in an orthopaedic hospital. Occup Med (Lond). 2005;55(6):498-500.

5. Sanders R, Koval KJ, DiPasquale T, Schmelling G, Stenzler S, Ross E. Exposure of the orthopaedic surgeon to radiation. J Bone Joint Surg Am. 1993;75(3):326-30.

6. Tsalafoutas IA, Tsapaki V, Kaliakmanis A, Pneumaticos S, Tsoronis F, Koulentianos ED, et al. Estimation of radiation doses to patients and surgeons from various fluoroscopically guided orthopaedic surgeries. Radiat Prot Dosimetry. 2008;128(1):112-9.
}

of the tibia. Comparatively, we needed 45 seconds for malleolar fractures, 41 seconds for osteosynthesis of the distal third of the radius with plate, 200 seconds for LIN of the femur, and 103 seconds for LIN of the tibia. Values two to three times lower than the study cited. We found studies with even greater times. Levin et $\mathrm{al}^{(7)}$ required, on average, 756 seconds of use of fluoroscopy to perform LIN of the femur, and 358 seconds for LIN of the tibia.

We find in the literature few studies that show times of fluoroscopy use similar to those in our work. Ricci et $\mathrm{al}^{(8)}$ required 113 seconds of use of image intensifier to perform LIN of the femur, and 82 seconds for LIN of the tibia. Hafez et $\mathrm{al}^{(9)}$ found in their study an average of 200 seconds in LIN of the femur, and 176 seconds in LIN of the tibia.

It is known that the radiation time necessary in an orthopedic surgical operation depends on various factors: type and difficulty of the surgical procedure, quality of the image intensifier device used, surgeon's experience, experience of the radiology technician who handles the image intensifier, and even the time of day the surgery is performed ${ }^{(8,10,11)}$.

\section{CONCLUSIONS}

Considering the 80 orthopedic surgeries investigated in this study, we found an average fluoroscopy time of 61 seconds. The operations that used the image intensifier most were proximal femoral epiphysiodesis (310 seconds) and those that used the locked intramedullary device, particularly femoral synthesis (200 seconds). Compared with the majority of studies found in the literature, we noted that ours needed less fluoroscopy time when performing orthopedic surgeries.

7. Levin PE, Schoen RW Jr, Browner BD. Radiation exposure to the surgeon during closed interlocking intramedullary nailing. J Bone Joint Surg Am. 1987;69(5):761-6.

8. Ricci WM, Gallagher B, Brandt A, Schwappach J, Tucker M, Leighton R. Is afterhours orthopaedic surgery associated with adverse outcomes? A prospective comparative study. J Bone Joint Surg Am. 2009;91(9):2067-72

9. Hafez MA, Smith RM, Matthews SJ, Kalap G, Sherman KP. Radiation exposure to the hands of orthopaedic surgeons: are we underestimating the risk?. Arch Orthop Trauma Surg. 2005;125(5):330-5

10. Blattert TR, Fill UA, Kunz E, Panzer W, Weckbach A, Regulla DF. Skill dependence of radiation exposure for the orthopaedic surgeon during interlocking nailing of long-bone shaft fractures: a clinical study. Arch Orthop Trauma Surg. 2004;124(10):659-64

11. Mesbahi A, Rouhani A. A study on the radiation dose of the orthopaedic surgeon and staff from a mini C-arm fluoroscopy unit. Radiat Prot Dosimetry. 2008;132(1):98-101. 